\title{
Diagnosis of "fizz-gas" and gas reservoirs in deep-water environment
}

De-hua Han, X RPL, Houston Unversity

Michael Batzle, Colorado School of Mine

\section{Introduction}

"Fizz-water" or "Fizz-gas" is a rather ill-defined and misused concept. For some, it refers to gas in solution with brine; for others, it is defined as small amounts of free gas phase. This small, uneconomic gas content then gives rise to seismic bright-spots or other Direct Hydrocarbon Indicators (DHIs). Unfortunately, it is often the culprit of choice when no other reason can be found. However, progress has been made in assessing the problem. We have systematically examined physical properties of fluid and rock, and fluid interaction with rock to examine gas saturation effect on acoustic velocities, especially in deepwater sands of the Gulf Mexico . Furthermore, we have reviewed the current AVO and Rock physics interpretation techniques to propose optimum DHIs. Several promising techniques of seismic evaluation of gas saturation are in development.

\section{Gas and water properties}

Han and Batzle (2003) have systematically studied gas effects on fluid modulus. Measured data show that dissolved gas has negligible effect on water velocity, modulus, and density. In addition, gas bubbles exsolving from either water or oil have only a small effect on bulk fluid properties at pressures higher than about $20 \mathrm{MPa}$ (about $3000 \mathrm{psi}$ ). Gas properties progressively transit to those of light oils with increasing pressure. Gas effects on fluid modulus depend on two factors: gas has to be in free phase and gas pressure has to be low (less than $20 \mathrm{MPa}$ ).

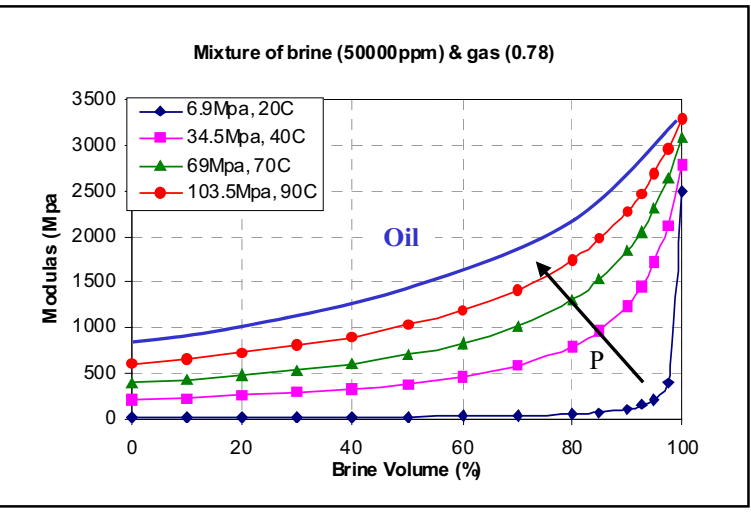

Figure 1. Modulus of Gas-water mixture depends on gas and water modulus and pressure and temperature conditions.
Figure 1 shows that modulus of gas-water mixture at isostress condition is calculated with Reuss bound (Wood, 1955) and mainly controlled by pressure. At low pressure (shallow depth $<2000 \mathrm{~m}$ ), gas modulus is much less than $0.1 \mathrm{GPa}$. Even few percent volume fraction of gas can drastically reduce the modulus of gas-water mixture. However, at a high pressure condition, modulus of gaswater mixture shows progressive decrease with increasing gas saturation and results in differentiable DHI attribute. Fluid modulus depends on composition, distribution and reservoir conditions, which are a result of complicated geological processes which form a reservoir.

Phenomenon associated with strong DHI anomalies for "fizz-gas "should correlate with both low fraction $(<30 \%)$ and low pressure $(<20 \mathrm{MPa})$ gas. The "fizz-gas" should also be correctly termed as "residual-gas".

\section{Fluid saturation effect}

Surface seismic data are a measure of impedance contrast of sediment (shale and sand) interface. Fluid saturation effects on seismic velocities can be described by the simplified Gassmann's equation for high porosity sands (Han and Batzle, 2004).

$$
\begin{aligned}
& K_{s}=K_{d}+\Delta K_{d} ; \quad \mu_{d}=\mu_{s} \\
& \Delta K_{d}=G(\phi) * K_{f}
\end{aligned}
$$

where subscript $\mathrm{d}$ and $\mathrm{s}$ are for dry and saturated rock respectively; $\mathrm{f}$ is for pore fluid and $\phi$ is porosity. The $G(\phi)$ is the gain function of dry rock frame given as

$$
G(\phi)=\left(1-K_{d} / K_{0}\right) / \phi
$$

The Gassmann's equation suggests that there is fluid effect on the bulk modulus but not on shear modulus. And the portion of fluid contribution into the bulk modulus is approximately proportional to pore fluid modulus $K_{f}$ and the gain function $G(\phi)$, which is a dry rock frame property. We rewrite the above equation with $\mathrm{P}$-wave modulus as

$$
\begin{aligned}
M & =\rho^{*} V_{P}^{2} \\
M_{s} & =K_{d}+4 / 3 * \mu_{d}+\Delta K_{d} \\
& =M_{d}+G(\phi) * K_{f}
\end{aligned}
$$




\section{Diagnostic "fizz-gas" and gas reservoirs}

The fluid effect on the P-wave modulus depends on sensitivity of $\mathrm{P}$-wave modulus $M_{s}$ to variation of fluid modulus $K_{f}$. The fluid saturated P-wave modulus $M_{s}$ is dependent on pore fluid modulus $K_{f}$ as well as dry frame Pwave modulus $M_{d}$ and dry frame gain function $G(\phi)$. In this relation, the $K_{d}$ and $\mu_{d}$ are correlated and constrained by $G(\phi)$ through the Gassmann's equation. To evaluate fluid saturation, we have to know both rock and fluid properties.

\section{The sensitivity of P-wave modulus to fluid modulus}

The minimum of $M_{s}$ of reservoir rock is equal to the $M_{d}$ with pore fluid modulus $K_{f}$ equals zero (vacuum) and the maximum of $M_{s}$ is assumed as that with (background) water saturation. Here, the modulus of water depends on salinity and reservoir conditions and assumed to be higher than that of hydrocarbon (it may not be true for a heavy oil reservoir). We define the relative sensitivity of P-wave modulus to pore fluid as

$$
\Delta_{f}=1-\left(M_{d}+G(\phi) * K_{f}\right) / M_{w}
$$

The maximum sensitivity is $\Delta_{\max }=1-M_{d} / M_{w}$. The sensitivity to differentiate fizz from gas reservoir

$$
\Delta_{f-g}=G(\phi) *\left(K_{f i z z}-K_{g}\right) / M_{w}
$$

To differentiate the "fizz" gas from gas reservoir it mainly depends on difference of the modulus of the "fizz" and gas fluids. The sensitivity also depends on the gain function and the P-wave modulus of background water zones.

\section{Gain function of deep-water unconsolidated sands}

Deep-water gas reservoirs in the Gulf of Mexico are often hosted in young, unconsolidated turbidite sands. We have measured velocities of core samples. The measured data suggest that the bulk modulus is very sensitive to water saturation at in situ conditions as shown in Figure 2. It is interesting that the calculated gain function based on measured data on those porous sands tend to approach a constant of 2.5 as shown in Figure 3. The Gain function tends to decrease with decreasing porosity and increasing cementation. Using the gain function, we can derive fluid modulus using the differencel of the fluid saturated and the dry modulus of rock.

$$
\begin{aligned}
& \Delta K_{d}=\Delta M_{d}=M_{s}-M_{d}=G(\phi) * K_{f} \\
& K_{f}=\Delta M_{d} / G(\phi)
\end{aligned}
$$

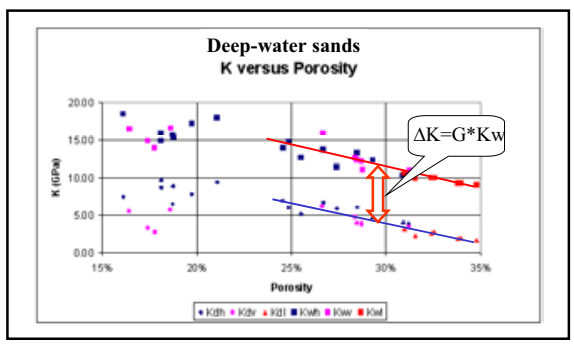

Figure 2. Measured dry and water saturated bulk modulus for deep-water sands show water saturation effect.

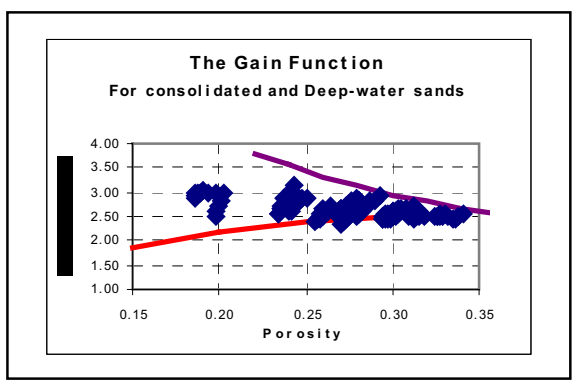

Figure 3. Gain function for deep-water sands

\section{Optimum hydrocarbon indicator}

Many indicators are now being developed to address residual-gas discrimination. Russell et al. (2003) summarized the DHI techniques associated with AVO technique. The indicators can usually be reduced to a form dependent on the difference between the compressional and shear impedances: $\mathrm{Z}_{\mathrm{p}}^{2}-\mathrm{CZ}_{\mathrm{s}}^{2}$, where $\mathrm{C}$ is a calibration constant. We (Batzle et al., 2001) have suggested that $\mathrm{C}$ should be equal to square of dry $\mathrm{Vp} / \mathrm{Vs}$ ratio. Goodway (1997) has suggested $\lambda-\mu$ method (equivalent $\mathrm{C}=2$ ) and Hadlin (2000) has adopted $\mathrm{K} / \mu=0.9$ (equavilent $\mathrm{C}=2.23$ ). Dillon et al. (2003) pointed out that the value of this constant $\mathrm{C}$ is important in maximizing the hydrocarbon discrimination, and is often larger than the values of $\mathrm{C}=2.33$ suggested by Batzle et al., (2001). For deep-water unconsolidated sand reservoirs, modulus and density of gas tend to be high, but can vary over a wide range. We actually have a chance to differentiate a gas reservoir from a residual-gas zone, if we can carefully calibrate the seismic parameters. Figure 4 shows relative attributes of "fizz-gas" and gas cases normalized by the values with water saturation. Attributes such as modulus $K$, fluid factor $\Delta K, \quad \lambda \rho, \quad \rho * \Delta K, \quad \rho^{*} K_{f}, \quad$ and $K_{f}, \quad$ illustrate significant differences between residual-gas and gas reservoirs. All these attribute show similar sensitivity and are mainly controlled by fluid modulus $K_{f}$. 


\section{Diagnostic "fizz-gas" and gas reservoirs}

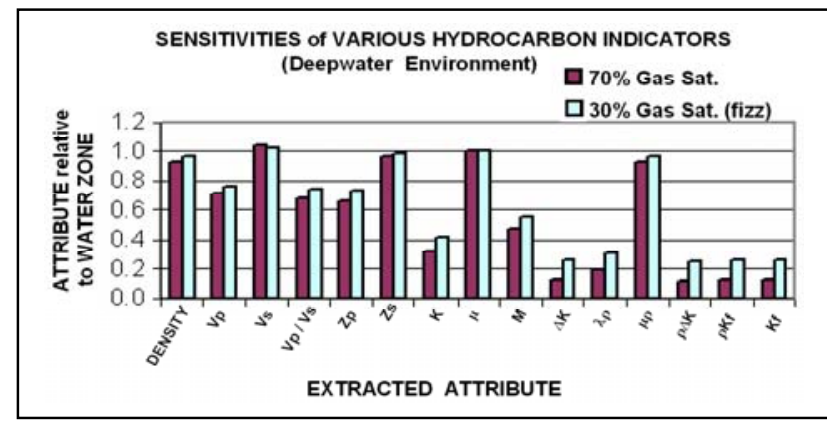

Figure 4. Sensitivity of 15 different hydrocarbon indicators in deep-water fizz and gas reservoirs.

In comparison to the shallow case, normal reflectivity appears to be the best residual-gas indicator as shown in Figure 5.

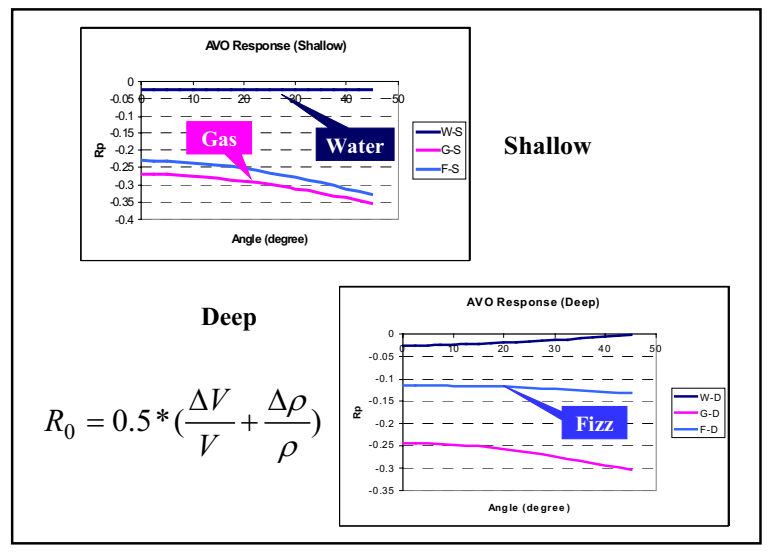

Figure 5. AVO responses for deep- and shallow sands.

We need better methods to calibrate seismic attributes not only on gas zones, but equally important brine zones to give us background calibration. Forward modeling, with accurate rock and fluid properties, and reservoir structure (include fluid distribution), is also a powerful tool to quantify hydrocarbon indicators.

However, in practice seismic attributes are not only affected by rock and fluid properties, but also by scattering and intrinsic dispersion and attenuation due to property heterogeneity and different frequencies (wavelength). Question is how we can separate the scaling effects to make sure that seismic attributes are proper to be used for rock and fluid property inversion.

\section{Scaling effects on seismic attribute}

Forward modeling often starts with well logging data, building synthetic seismogram to compare with near-by seismic gather. Seismic parameters such as velocity can be significantly affected by scale dependent heterogeneity. For two material layer model, effective velocity (as well as impedance) can vary in wide range depending on wavelength/layer thickness ratio as shown in Figure 6 (Mavko et. al., 1998).

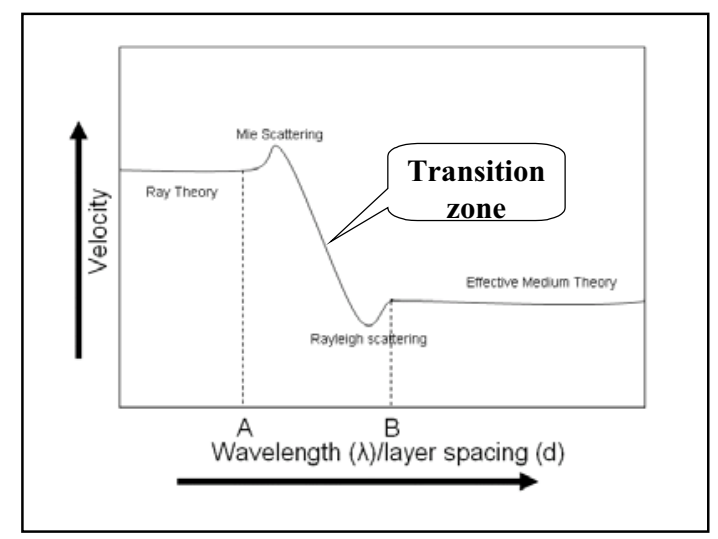

Figure 6. Velocity dispersion due to layer structure

Unfortunately, seismic properties in sedimentary basin are often located in the transition zone, and seismic attributes are hard to obtain. For example, AVO attributes (Figure 7) can be affected by both fluid properties and thin layer tuning.

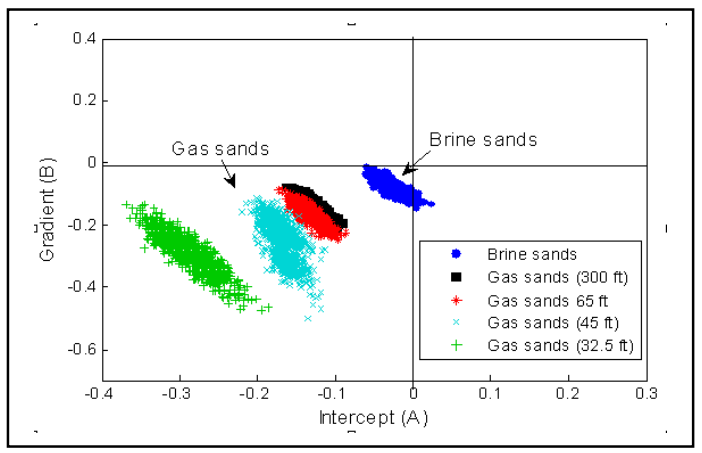

Figure 7. AVO attributes A and B for typical deep-water sands with different thin layer tuning effect.

Therefore, we may have to apply wave propagation model to include scattering dispersion and attenuation effects. Furthermore, we may have to develop inelastic model to include intrinsic dispersion and attenuation into synthetic seismogram to evaluate the seismic wave propagation effects on seismic attribute. Eventually, we may be able to separate the wave propagation effects on seismic attributes, before which can be then be used for quantitative evaluation of gas saturation.

\section{Residual gas reservoirs}

Forming a gas reservoir is a result of many geological processing, such as hydrocarbon resources, maturation, migration (gas resolves in, or exsolves out of water and hydrocarbon, equilibrium between capillary trap, gravity force and chemical diffusion), trap and accumulation (seal 


\section{Diagnostic "fizz-gas" and gas reservoirs}

and leak equilibrium) with back ground of sedimentary processing. The gas distribution can be ranged in many phases from gas layer with good trap, or poor accumulation with leaking trap, or continue seep to surface as gas chimney. Each phase can be in different scale from microsize in pore space to mega-size in hundred meters. Residual gas reservoir often associates to leaking trap or poor resources.

We are suspicious that very thin gas layer (a foot thick?) along top of lithology interface may generate shape impedance contrast, which may be able to block seismic wave to generate a bright reflection, but invisible for conventional log tools.

\section{Potential techniques}

In the future, attenuation (1/Q), or frequency content, might prove a helpful attribute as revealed in measured data shown in Figure 8 (Kumar, et al., 2003). Less is understood of $1 / \mathrm{Q}$, but several researchers recently have reported success in using frequency content as a discriminator of hydrocarbons. In this case, fluid properties, distributions, and mobility all contribute. However, the in situ fluid distribution and mobility are often unknown. In addition, even the controlling mechanisms of attenuation are not well understood.

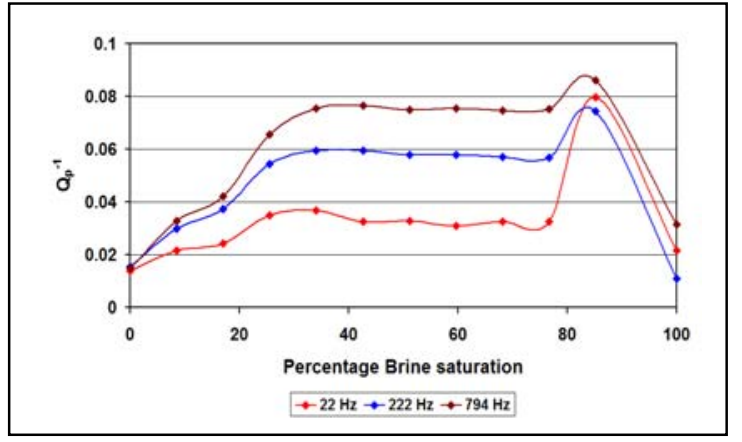

Figure 8. Measured attenuation versus gas saturation reveals that low gas saturation may be related to high attenuation.

\section{Acknowledgments}

We would like to recognize the support from Department of Energy Award DE-FC26-02NT15342 and the 'Fluids/DHI Consortium' by our corporate sponsors.

\section{Reference}

Batzle, M., Han, D., and Hoffman, R., 2001, Optimal hydrocarbon indicators: Presented at the 71 Ann. Internat. Mtg., Soc. Expl. Geophys., Expended Abstracts, Dillon, L., Schwedersky, G., Vasquez, G., Velloso, R., and Nunes, C., 2003, A multiscale DHI elastic attribute evaluation. The Leading Edge, 23, 1024-1029.

Goodway, B., Chen, T., and Downton, J., 1997, Improved AVO fluid detection and lithology discrimination using lame petrophysical parameters: $\lambda \rho, \mu \rho \& \lambda / \mu$ fluid stock, from $\mathrm{P}$ and S-wave inversions: Presented at the 67 Ann. Internat. Mtg., Soc. Expl. Geophys., Expended Abstracts, 183-186.

Harding, K., 2000, Pore space modulusand extraction using AVO: 70 th Ann. Internat. Mtg, SEG., Expanded Abstract, 170-173.

Han, D., and Batzle, M., 2002, Fizz water and low gas saturated reservoirs: The Leading Edge, 21, 395-398.

Han, D., and Batzle, M., 2004, Gassmann's equation and fluid saturation effects on seismic velocities: Geophysics, P-398-405

Kumar, G., Batzle, M. and Hofmann, R., 2003, Effect of fluids on attenuation of elastic waves, 73rd Ann. Internat. Mtg.: Soc. of Expl. Geophys., 1592-1595.

Mavko, G., Mukerji, T., and Dvorkin, J., 1998, The rock physics handbook-Tool for seismic analysis in porous media: Cambridge Univ. Press.

Russell, B., Hedlin, K., Hilterman, F. J., and Lines, L., R., 2003, Fluid-property discrimination with AVO: A BiotGassmann perspective: Geophysics, 68, 29-39.

Wood, A. W., 1955, A Textbook of Sound. The MacMillan Co., New yark, 360 PP. 


\section{EDITED REFERENCES}

Note: This reference list is a copy-edited version of the reference list submitted by the author. Reference lists for the 2005 SEG Technical Program Expanded Abstracts have been copy edited so that references provided with the online metadata for each paper will achieve a high degree of linking to cited sources that appear on the Web.

Diagnosis of "fizz-gas" and gas reservoirs in deep-water environment

\section{REFERENCES}

Batzle, M., D. Han, and R. Hofmann, 2001, Optimal hydrocarbon indicators: 71st Annual International Meeting, SEG, Expanded Abstracts, 1697-1700.

Dillon, L., G. Schwedersky, G. Vasquez, R. Velloso, and C. Nunes, 2003, A multiscale DHI elastic attribute evaluation: The Leading Edge, 23, 1024-1029.

Goodway, B., T. Chen, and J. Downton, 1997, Improved AVO fluid detection and lithology discrimination using lame petrophysical parameters: $\lambda \rho, \mu \rho \& \lambda / \mu$ fluid stock, from P and S-wave inversions: 67th Annual International Meeting, SEG, Expanded Abstracts, 183-186.

Han, D., and M. Batzle, 2002, Fizz water and low gas saturated reservoirs: The Leading Edge, 21, 395-398.

Han, D., and M. Batzle, 2004, Gassmann's equation and fluid saturation effects on seismic velocities: Geophysics, 69, 398-405.

Hedlin, K., 2000, Pore space modulus and extraction using AVO: 70th Annual International Meeting, SEG, Expanded Abstracts, 170-173.

Kumar, G., M. Batzle, and R. Hofmann, 2003, Effect of fluids on attenuation of elastic waves: 73rd Annual International Meeting, SEG, Expanded Abstracts, 1592-1595.

Mavko, G., T. Mukerji, and J. Dvorkin, 1998, The rock physics handbook-Tool for seismic analysis in porous media: Cambridge University Press.

Russell, B., K. Hedlin, F. J. Hilterman, and L. R. Lines, 2003, Fluid-property discrimination with AVO: A Biot-Gassmann perspective: Geophysics, 68, 29-39.

Wood, A. W., 1955, A textbook of sound: The MacMillan Co. 